\section{$\S 2 . \quad$ Properties of Ballooning Modes in the Planar Axis Heliotron Configurations with a Large Shafranov Shift}

\author{
Nakajima, N., Hudson, R.S. (PPPL), Hegna, C.C. \\ (Wisconsin U.)
}

The research on the ballooning modes in the planar axis heliotron configurations allowing a large Shafranov shift like LHD are summarized systematically, with particular attention given to the high- $\beta$ MHD equilibria in the inward-shifted LHD configurations. The relation of the quasi-modes to physical modes, the relation between ballooning modes and interchange modes, and also the effects of the boundary condition of the perturbations on the pressure-driven modes are clarified [1].

In the three-dimensional magnetic confinement configurations, the results of local mode analyses of the ballooning modes (quasi-modes) in the covering space along the magnetic field line can not be directly connected to those of the global mode analyses of the ballooning modes in the configuration space (physical modes), because the rule of superposition of the quasi-modes is not allowed because of the lack of symmetry. In other words, the eikonal $S(\psi, \alpha)$, where $\psi$ and $\alpha$ are the lavel of the flux surfaces and magnetic field lines, respectively, can not be determined, so that the phase relation of the quasi-modes with same eigenvalue is not established in superposing the quasi-modes. However, in planar axis heliotron configurations with a large Shafranov shift, a qualitative relation has been established that the quasi-ballooning modes in the covering space can be connected to physical ballooning modes in the configuration space. Such a relation comes from the topological structure of the level surfaces of the eigenvalues in the $\left(\psi, \theta_{k}, \alpha\right)$ space, where $\psi, \theta_{k}$, and $\alpha$ are the label of the flux surfaces, the radial wave number, and the label of the magnetic field line on a flux surface, respectively. In the three-dimensional magnetic configurations, two types of level surafaces exist in the $\left(\psi, \theta_{k}, \alpha\right)$ space. One is the topologically spheroidal level surfaces, and the other is topologically cylindrical level surfaces. The former's quasimodes correspond to the three-dimensional ballooning modes inherent to three-dimensional configurations consisting of both relatively high poloidal and toroidal mode numbers through strong poloidal and toroidal mode coupling. The latter's quasi-modes correspond to the two-dimensional ballooning modes with fairly weak toroidal mode couplings, which are similar to those in the axisymmetric configurations, and to interchange modes. In the Mercier stable MHD equilibria, only three-dimensional ballooning modes inherent to three-dimensional configurations might be excited. In the Mercier unstable MHD equilibria, not only three-dimensional ballooning modes as well as in the Mercier stable equilibria but also two-dimensional ballooning modes and/or interchange modes might be excited. Since the latter has the topologically cylindrical level surfaces of eigenvalues of quasi-modes in the $\left(\psi, \theta_{k}, \alpha\right)$ space, the approximate quantization condition connecting the quasi-modes in the covering space to physical modes in the configuration space is applicable to them. Note that the quantization condition can be applicable to both two-dimensional ballooning modes and interchange modes. Two-dimensional ballooning modes and interchange modes have smaller growth rates than those of three-dimensional ballooning modes. Theer are two interesting points. By using the equilibrium profile variation method, it is shown that recently achieved high- $\beta$ MHD equilibria in the inwardshifted LHD configurations stay in the second stable state in the plasma core and stay near the marginally stable state in the plasma periphery. It should be clarified why the plasma core is easy to stay the second stability, and whether the experimentally suggested pressure profiles result from the ballooning instabilities or not.

Another interesting point is whether $3 \mathrm{D}$ ballooning modes are systematically distinguished or not. There is a case that the $3 \mathrm{D}$ ballooning mode has one radial node $\left(N_{r}=1\right)$. Since $3 \mathrm{D}$ ballooning modes consist of the superposition of the 2D-like ballooning modes, if there is a rule in the superposition, then 3D ballooning modes might be systematically distinguished by the radial node numbers $N_{r}$ and the behavior of the poloidal localization, for example, the number of the poloidal nodes created by the localization in the flux tubes. This point is associated with the integrability condition of the two degree of freedom Hamiltonian system related to the ray equations of the eikonal.

[1] N.NAKAJIMA, S.R.HUDSON, and C.C.HEGNA, Fusion Sci. and Tech. (accepted). 